\title{
The rosette-forming glioneuronal tumor mimicked cerebral cysticercosis: a case report
}

\author{
Dan Zhu ${ }^{1} \cdot$ Ailan Cheng ${ }^{1} \cdot$ Nickita T. L. Benons ${ }^{2} \cdot$ Shuguang $\mathrm{Chu}^{1}$ \\ Received: 10 January 2021 / Accepted: 16 March 2021 / Published online: 20 May 2021 \\ (C) The Author(s) 2021
}

\begin{abstract}
Introduction Rosette-forming glioneuronal tumor (RGNT) is a rare variety of slow growing mixed glioneuronal tumor involving primarily fourth ventricular region. This is a comprehensive analysis of a 22-year-old woman with RGNT composed of mainly cystic components. In addition, the case showed multiple lesions located in brain parenchyma which mimicked cerebral cysticercosis. Here, we analyzed this case and listed some characteristics of RGNTs in reported literature which occurring in atypical locations for further understanding it.

Case report A 22-year-old woman presented with a history of transient dizziness, nausea, and vomiting. Magnetic resonance imaging (MRI) showed multiple cystic lesions in brain parenchyma and then the patient was diagnosed with cerebral cysticercosis possibility. Empirical anti-infective therapy in addition to a follow-up post 2 weeks of MRI examination showed the lesions unchanged. Finally, a biopsy of the right cerebellar hemisphere lesions verified RGNT.

Conclusion RGNT is an uncommon tumor classified as grade I glioma by World Health Organization (WHO) with slightly longer course. The imaging findings of RGNT are not specific especially in atypical areas. RGNT is rare, but we should also consider the possibility in diagnosis and differential diagnosis.
\end{abstract}

Keywords Rosette-forming glioneuronal tumor $\cdot$ Cerebral cysticercosis $\cdot$ Magnetic resonance imaging $\cdot$ Hemorrhage

\section{Introduction}

The rosette-forming glioneuronal tumor (RGNT) was first described by Komori et al. in 2002. As it was initially thought as dysembryoplastic neuroepithelial tumor (DNT) of the cerebellum [1]. In 2007, it was classified as grade I glioma by World Health Organization (WHO). RGNT occurs most often in

Dan Zhu and Ailan Cheng contributed equally to this work and should be considered co-first authors.

Shuguang Chu

chushu1018@hotmail.com

Dan Zhu

18895338344@163.com

Ailan Cheng

chengailan2009@163.com

1 Department of Radiology, Shanghai East Hospital, Tongji University, No. 150 Jimo Road, Pudong New Area, Shanghai 200120, China

2 Tongji University, No. 1239 Siping Road, Yangpu District, Shanghai 200092, China young women with mean age of onset at 23.57 years [2]. There are few literatures regarding the imaging features and prognosis of RGNT. For most of the literatures on RGNT are case reports. RGNT is most commonly located in the fourth ventricle; however, recent reports demonstrated that RGNT can also occur at sites outside its usual locations. The lesions are mostly comprised of cystic-solid or solid, and the solid components present heterogenous enhancement. Here, we describe a rare case of a 22-year-old woman with RGNT in bilateral cerebellar hemisphere, brain stem, and left thalamus who was misdiagnosed as cerebral cysticercosis before biopsy.

\section{Case report}

A 22-year-old woman presented with a history of transient dizziness, nausea, and vomiting. No neurological deficits were apparent; however, on further evaluation, initially with computed tomography (CT) scan, revealed multiple cystic hypo-dense mass lesions in bilateral cerebellar hemisphere, brain stem, and left thalamus with unclear boundary (Fig. 1a-c). Magnetic 

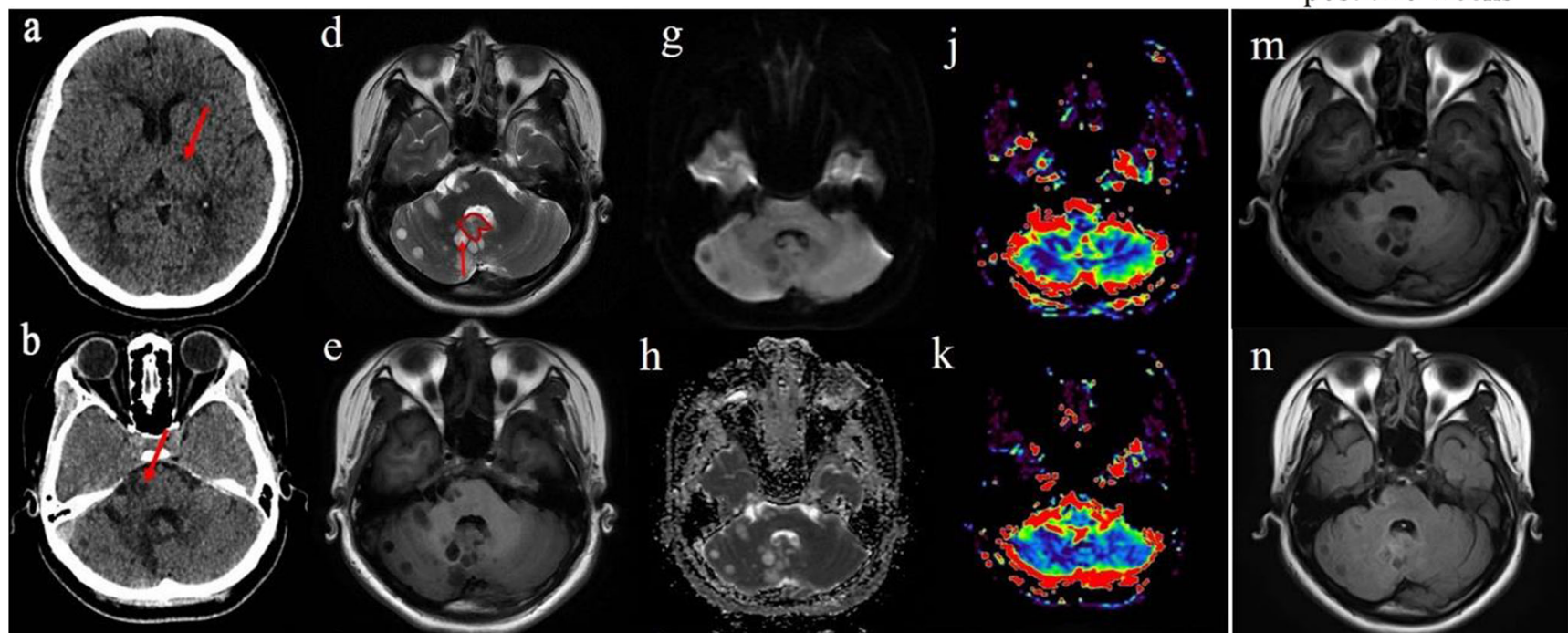

k
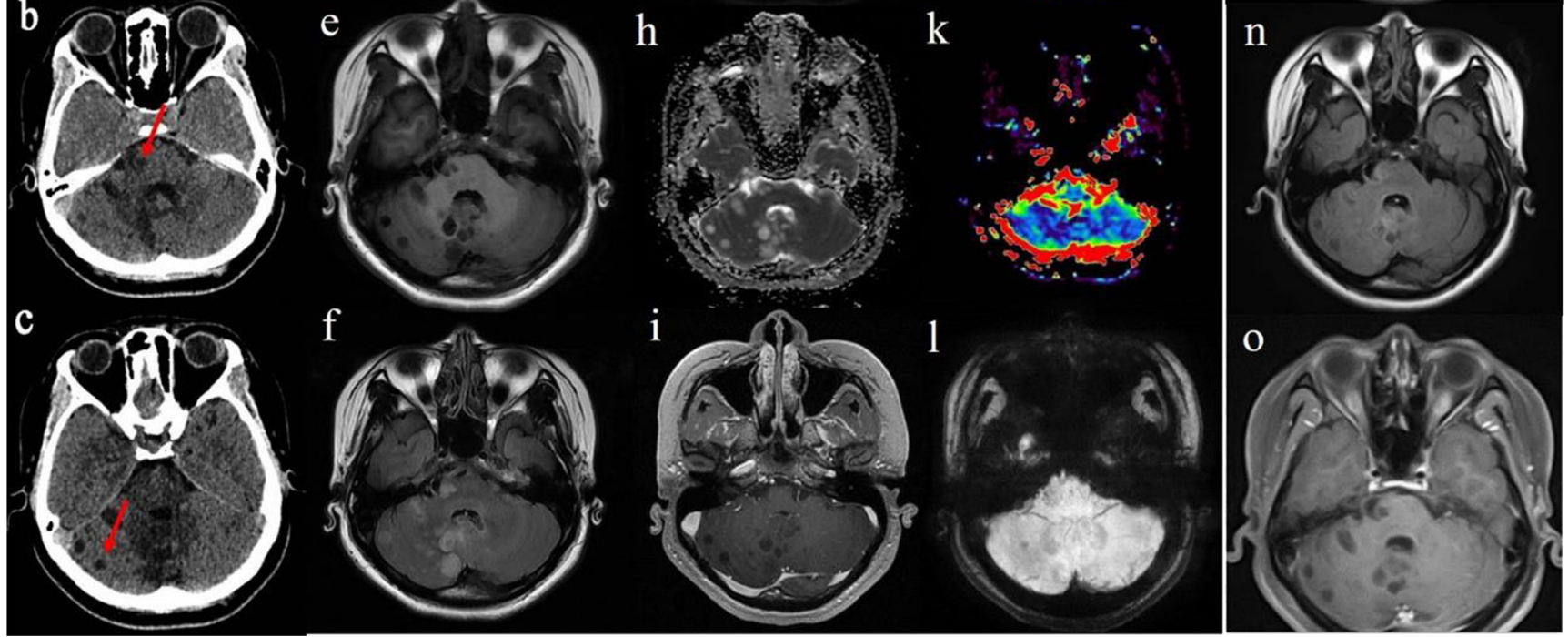

Fig. 1 a-c CT findings show the tumors in left thalamus, brain stem, and bilateral cerebellar hemisphere (red arrow). d-I MRI findings. The lesions in bilateral cerebellar hemisphere. $\mathbf{d}$ Hyper-intense in T2-weighted image and small nodule-like higher signal (red arrow) along with circled solid component. e Hypo-intense in T1-weighted image. f T2 flair image showed iso-hyper intense. g Hypo-intense in DWI. h Hyper-intense in
ADC. i No obvious enhancement majority and minority presented as mild annular enhancement. $\mathbf{j}-\mathbf{k}$ Perfusion-weighted imaging color map, decreased regional cerebral blood flow, and regional cerebral blood volume. I minor hemorrhage in the lesions. I SWI showed minor hemorrhage. $\mathbf{m}-\mathbf{0}$ MRI findings for post 2 weeks. $\mathbf{m}-\mathbf{o}$ Corresponding T1WI, T2 flair, and T1WI enhancement, no obvious changes compared with before resonance imaging (MRI) confirmed these lesions presented as hyper-intense in axial T2-weighted images and hypo-intense in axial T1-weighted images (Fig. 1d, e). The solid components were visible in some of the lesions in axial T2-weighted images (Fig. 1d). In addition, axial T2 FLAIR revealed isohyperintense (Fig. 1f). Diffusion-weighted imaging (DWI) and apparent diffusion coefficient (ADC) showed no restricted diffusion (Fig. 1g, h). After contrast, one of the tumors showed mild peripheral enhancement, while others presented no enhancement (Fig. 1i), and small nodule-like higher signals in T2-weighted images were present (Fig. 1d). While, on perfusion-weighted imaging, the lesions were hypo-perfused (Fig. 1j, k).

Based on the above radiological findings, the woman was initially diagnosed with cerebral cysticercosis most possibly. Naturally, primary tumors of the central nervous system and metastatic tumors were differential diagnoses. Subsequently, she was admitted to the infectious diseases department to conduct empirical anti-infective therapy in addition to a follow-up post 2 weeks of MRI examination. Over the course of 2 weeks, she underwent further laboratory examinations, including serologies (specifically enzyme-linked immunotransfer blots (EITBs)); however, the results were negative. Meanwhile, eosinophilic cells and lymphocytes were neither found in cerebrospinal fluid (CSF). Other parasite antibodies were not discovered either. Most importantly, there were no significant changes in the MRI findings after 15 days (Fig. $1 \mathrm{~m}-\mathrm{o}$ ). Finally, to determine an ultimate diagnosis, she underwent a biopsy of the right cerebellar hemisphere lesions in which the histopathological results confirmed a WHO grade I RGNT ultimately. Microscopically, the tumors showed that small round nuclear tumor cells were distributed in a network and arranged into a chrysanthemum-shaped cluster surrounding the nerve with single permutation. Synaptophysin immunopositivity in the pericapillary area of a perivascular pseudorosette was shown, along with scattered neurons in the focal areas were also visible. In addition, glial fibrillary acidic protein (GFAP) in the astrocytic component of the tumor was diffuse distribution. (Fig. 2a-e). 
Fig. 2 a-d Pathological pictures of the tumor. a Glioneuronal tumor with glial and neurocytic components. b Neurocytic rosette: small round nuclear tumor cells distributed in a network and a ring-like array of neurocytic tumor cell nuclei around an eosinophilic neuropil core (blue arrow) (hematoxylin-eosin: $a \times 200$; b×400). c, d Synaptophysin immunopositivity in the pericapillary area of a perivascular pseudorosette (red arrow) $(c \times 200 ; d \times 400)$. Diffuse positivity for glial fibrillary acidic protein (GFAP) in the glial component of the tumor $(\mathrm{e} \times 200)$
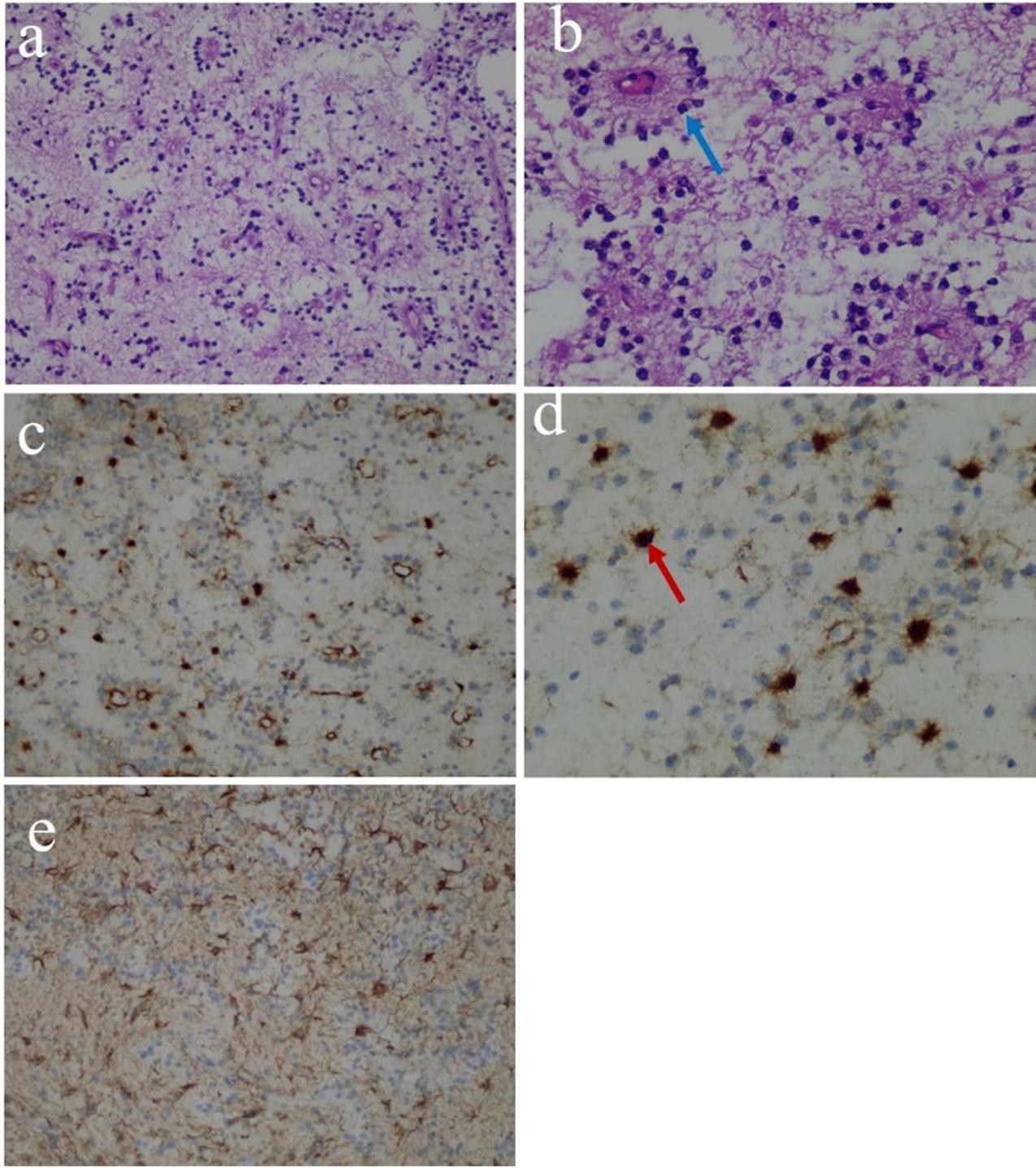

\section{Discussion}

RGNT is an unusual disease, and it is considered an independent entity of glioma, which is categorized as grade I by World Health Organization (WHO) due to its characteristics of containing both neural and glial components [3]. It is generally considered to be benign, but there have been reports that some can be invasive [4]. The disease was initially thought to only occur in the fourth ventricle, and the typical imaging characteristics are mid-line lesions which appeared in the fourth ventricle and extended to adjacent structures [5]. On MRI, RGNT typical imaging findings are relatively well circumscribed, with both solid and cystic components with T1-hypo-intense and T2-hyper-intense located in or around the fourth ventricle. Gadolinium-based contrast enhancement could show variable or no enhancement, but with increasing reports of the disease, other positions have also been reported, including the pineal region, pons, thalamus, spinal cord, optic chiasm, cerebellar hemisphere, optic pathway, lateral ventricle, septum pellucidum, cerebellar vermis, and even temporal lobe [6-9].

With regards to the English literature through a comprehensive search of Web of Science and PubMed using the search term "the rosette-forming glioneuronal tumor" nearly a decade, more than 100 articles have been published to date. After full text screening, excluding articles that were less relevant to the characteristics of RGNTs, nearly 70 articles were included by December 2020 finally. In general, 101 cases of RGNTs were reported located in the fourth ventricle, while 51 cases were located in atypical site. However, the imaging manifestations of RGNTs occurring outside the fourth ventricle are not specific; so, they are often misdiagnosed. Here, the characteristics of RGNTs located outside the fourth ventricle in 51 published cases were listed (Table 1). The lesions can be solid-cystic, cystic, or simple solid, and generally, the former is the most common. The average age of these published cases 


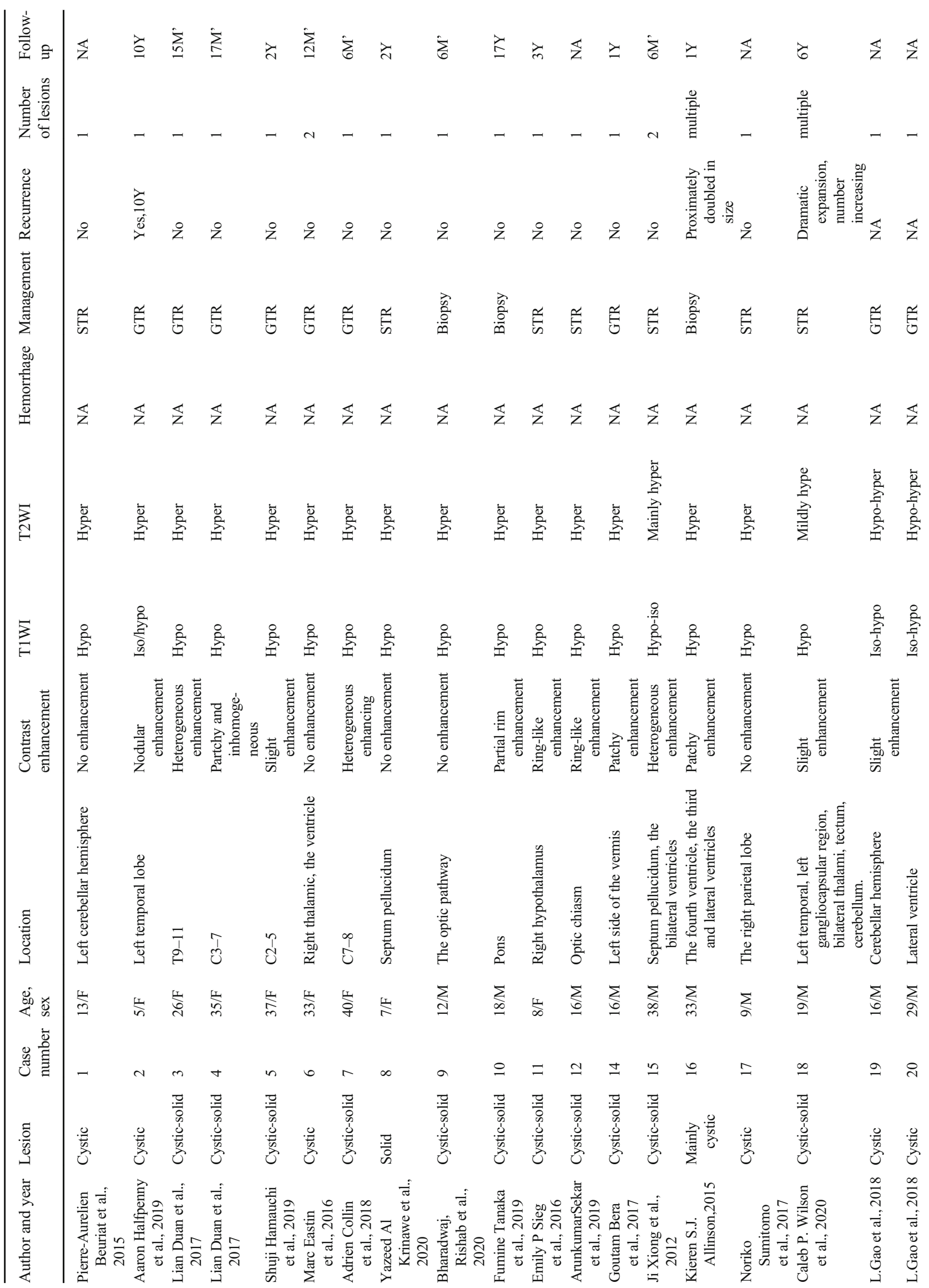




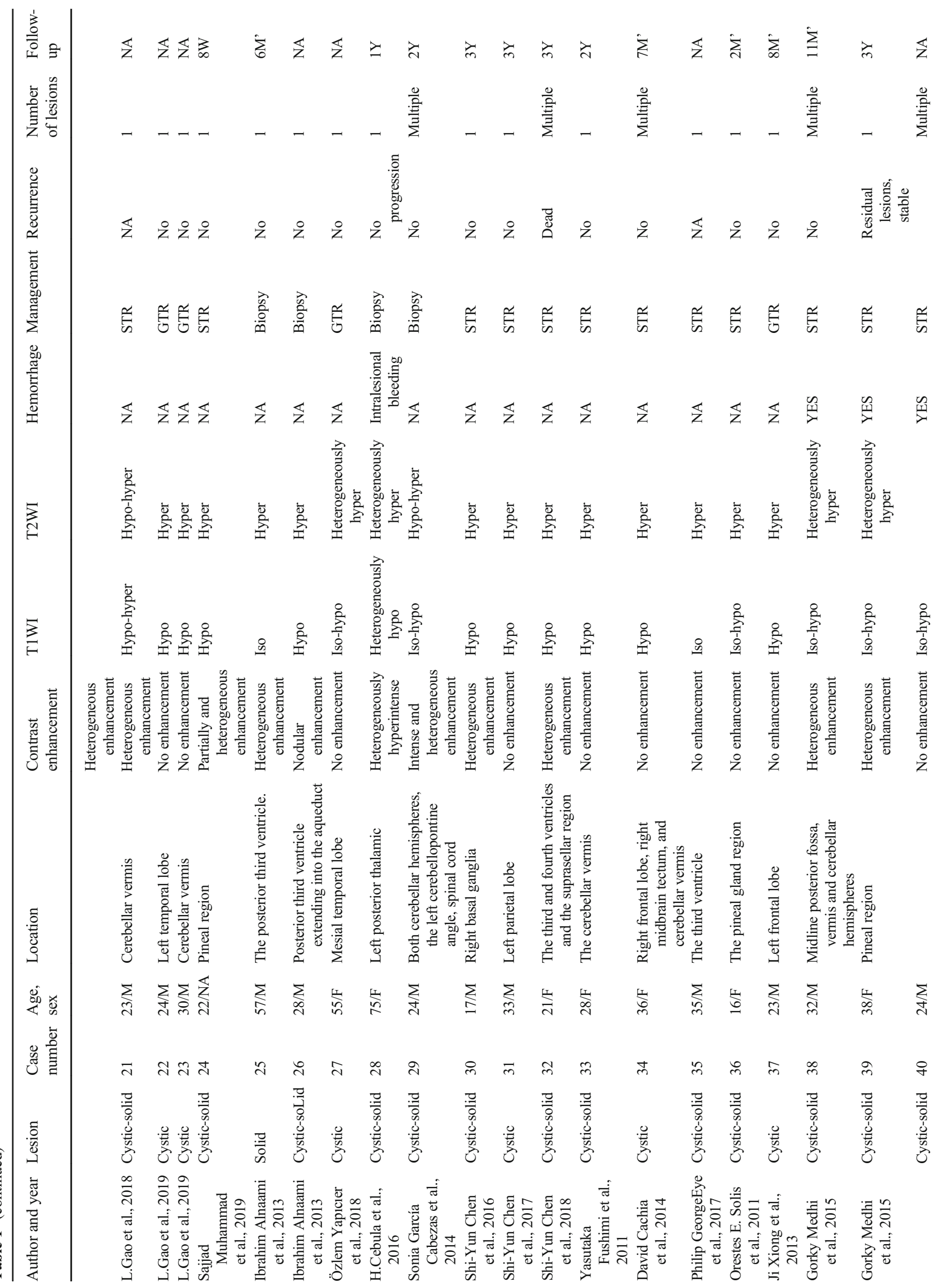




\begin{tabular}{|c|c|c|c|c|c|c|c|c|c|c|c|c|}
\hline & $\sum_{m}^{i}$ & $\grave{\xi}$ & $\overleftrightarrow{z}$ & $\underset{\dot{\sigma}}{\grave{r}}$ & $\underset{\underset{J}{J}}{ঙ}$ & $\sum_{\alpha}$ & 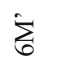 & $\bar{\delta}$ & $\overleftrightarrow{z}$ & $\overleftrightarrow{z}$ & $\sum_{-m}^{\bar{n}}$ & $\sum_{a}$ \\
\hline & - & $\begin{array}{l}\frac{0}{\mathscr{Z}} \\
\frac{E^{2}}{\Sigma}\end{array}$ & - & - & 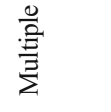 & - & - & - & 该 & - & - & - \\
\hline 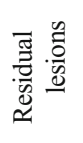 & 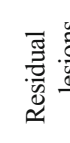 & 竞 & $\overleftrightarrow{z}$ & $\stackrel{\circ}{z}$ & $\stackrel{\circ}{z}$ & $\begin{array}{l}\sum_{\alpha} \\
\text { yे }\end{array}$ & 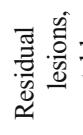 & $\frac{0}{0}$ & $\begin{array}{l}\frac{0}{\pi / \pi} \\
\frac{\pi}{\tilde{n}}\end{array}$ & $\overleftrightarrow{z}$ & 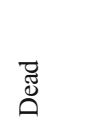 & $\stackrel{\circ}{z}$ \\
\hline & 苞 & 苞 & 苞 & 焗 & 苞 & 荌 & $\stackrel{\cong}{\mathscr{n}}$ & $\begin{array}{l}\overrightarrow{\mathrm{d}} \\
\stackrel{0}{0} \\
\overrightarrow{0}\end{array}$ & 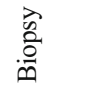 & 总 & $\stackrel{\mathscr{E}}{\mathrm{n}}$ & 苞 \\
\hline & $\stackrel{\circ}{z}$ & 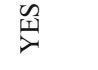 & $\mathbb{z}$ & $\overleftrightarrow{z}$ & $\overleftrightarrow{z}$ & $\mathbb{z}$ & $\overleftrightarrow{z}$ & $\overleftrightarrow{z}$ & $\stackrel{\mathscr{c}}{\underline{\lambda}}$ & $\overleftrightarrow{z}$ & $\overleftrightarrow{z}$ & $\overleftrightarrow{z}$ \\
\hline 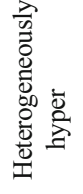 & 总 & 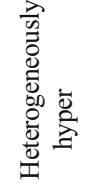 & 总 & 总 & 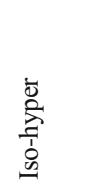 & 总 & 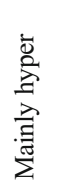 & 总 & 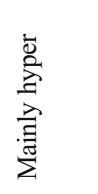 & 总 & 总 & 总 \\
\hline & 善 & 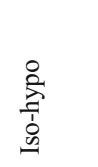 & 离 & 㤀 & 离 & 兽 & 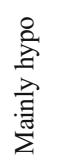 & 商 & 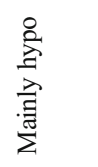 & 商 & 离 & 产 \\
\hline & 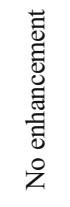 & 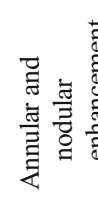 & 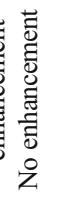 & 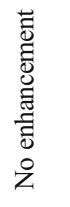 & 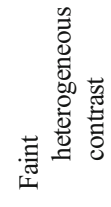 & 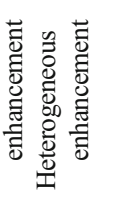 & 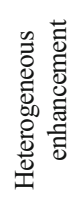 & 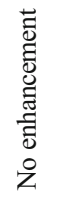 & 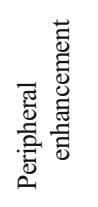 & 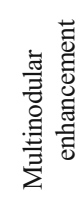 & 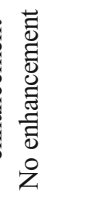 & 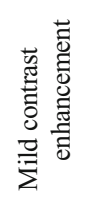 \\
\hline 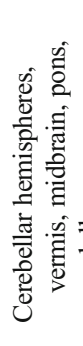 & 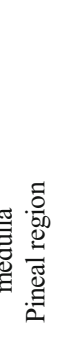 & 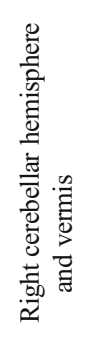 & 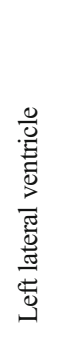 & 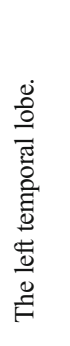 & 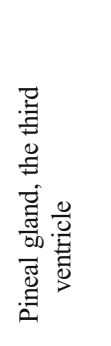 & 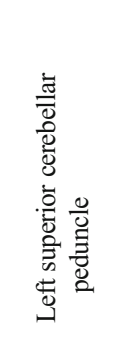 & 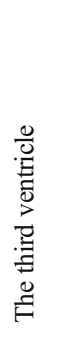 & 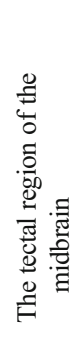 & 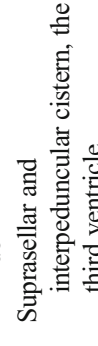 & 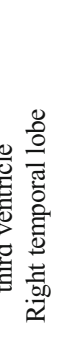 & 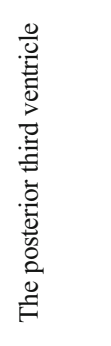 & 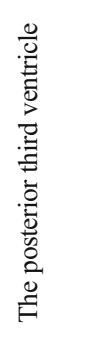 \\
\hline & $\sum_{\beth}$ & 㟶 & 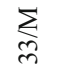 & 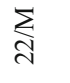 & $\underset{\text { j }}{\Sigma}$ & $\underset{\infty}{ \pm}$ & $\sum_{\forall}$ & $\stackrel{\underline{L}}{\underline{6}}$ & $\sum_{\leq}$ & $\stackrel{\mathrm{t}}{\mathrm{E}}$ & $\sum_{i}$ & $\sum_{\beth}$ \\
\hline & $\bar{\forall}$ & F & $\mathscr{F}$ & F & rq & fo & F & $\stackrel{\infty}{+}$ & g & in & $\vec{n}$ & $\approx$ \\
\hline & 苟 & 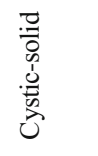 & 总 & 密 & 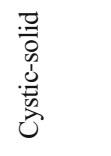 & 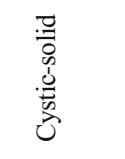 & 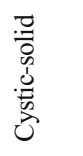 & 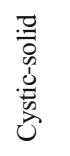 & 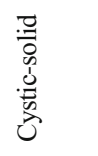 & 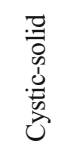 & 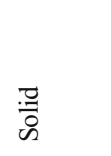 & 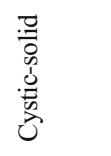 \\
\hline 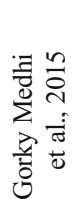 & 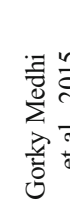 & 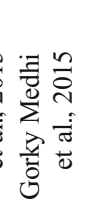 & 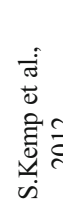 & 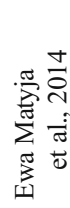 & 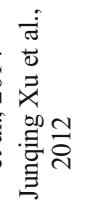 & 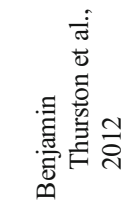 & 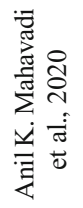 & 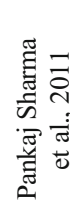 & 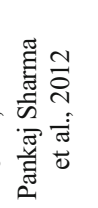 & 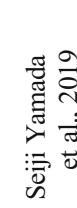 & 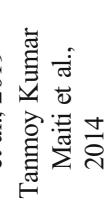 & 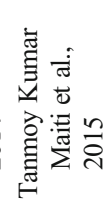 \\
\hline
\end{tabular}


is 38 years old. Hemorrhage is rare in RGNTs, and only six cases presented positive for bleeding. Management of RGNTs has been accordant with the literatures. Surgery remains the primary treatment option, with gross total resection (GTR) recommended and subtotal resection (STR) as alternatives. The prognosis of RGNT is generally good, and recurrence is uncommon with a total of 4 cases recrudesced of the 51 cases. However, two patients died of these presented cases. Most of the tumors were single lesions with only 10 cases showed multiple lesions.

In summary, the misdiagnosis in the above case reflects how RGNT is under-emphasized and poorly researched. Thus, based on the analysis of the present case and limited data available from review of literature, we propose that the following two aspects may have contributed significantly in misdiagnosing RGNT. Firstly, the case we highlighted occurred in the brain parenchyma, while more than $69.7 \%$ of previously reported RGNTs involve the fourth ventricle [3]. And multiple lesions involving the bilateral cerebellar hemisphere, brain stem, and left thalamus at the same time are rarely reported. Secondly, the imaging findings of this case overlapped with cerebral cysticercosis. As we all know, cerebral cysticercosis is the most common parasitic disease of the central nervous system (CNS). The imaging manifestations of the parenchymal active phase are multiple cystic lesions. The enhancement is not obvious and the hypo-perfusion on perfusion imaging. For the above reasons, the tumors were misdiagnosed as cerebral cysticercosis deservedly.

Admittedly, there are distinct radiological signs which highlight uncertainties regarding the previous diagnosis: most notably include tiny spot-like hypo-intense in bilateral cerebellar hemisphere on the susceptibility weighted imaging (SWI) (Fig. 11). After excluding tiny calcifications on CT (Fig. 1c), it can be assumed that there is minor hemorrhage within the lesions. Hemorrhage is almost invisible in cerebral cysticercosis, although there were also few reports of hemorrhages in RGNT. Two possible reasons may account for the latter. One being that the SWI sequence is rarely a routine sequence, and minor hemorrhages in many reported cases may go undetected because they are difficult to show on other sequences in MRI. On the other hand, it is generally assumed that RGNT is a benign tumor, microvascular proliferation is rare; therefore, the hemorrhages are infrequent as well. The evidence between microvascular endothelial proliferation and hemorrhages has been documented in RGNT [10, 11]. L. Gao et al. reported several cases of RGNTs with intratumoral hemorrhage in 2017. They summarized that intratumoral hemorrhage was one of the additional indications to the diagnosis of RGNT. Other indications included "green bell pepper sign," CSF dissemination, and multiple satellite lesions. Medhi et al. also summed up that hemorrhage and CSF dissemination may be the characteristics of RGNT through the summary of 7 cases [12]. In our case, intratumoral hemorrhage and multiple satellite lesions are consistent with their conclusions. Therefore, the above signs may aid in diagnosing RGNTs. However, whether this microvascular proliferation and intratumoral hemorrhage are related to prognosis needs further research.

\section{Conclusion}

RGNT is an uncommon low-grade neuroglial tumor which is generally considered benign with slightly longer course [2]. Headache is the most recorded common symptom. Histopathologically, RGNT consists of two components: a neurocytic component that forms rosettes, and an astrocytic component that resembles a pilocytic astrocytoma [13]. Through the case we reported, we have discovered that RGNT can be multiple cystic lesions, and the brain parenchyma can be the major affected areas. The intratumoral hemorrhage shown by SWI sequence may have some significance for our diagnosis of it. In conclusion, RGNT often presents significant diagnostic dilemma, and hence, further knowledge of this tumor is essential as they are relatively slow growing and exhibit benign histological characteristics.

\section{Declarations}

Conflict of interest The authors declare no competing interests.

Ethical approval This article does not contain any studies with humans.

Informed consent We would like to state that the informed consent has been obtained from the patient.

Open Access This article is licensed under a Creative Commons Attribution 4.0 International License, which permits use, sharing, adaptation, distribution and reproduction in any medium or format, as long as you give appropriate credit to the original author(s) and the source, provide a link to the Creative Commons licence, and indicate if changes were made. The images or other third party material in this article are included in the article's Creative Commons licence, unless indicated otherwise in a credit line to the material. If material is not included in the article's Creative Commons licence and your intended use is not permitted by statutory regulation or exceeds the permitted use, you will need to obtain permission directly from the copyright holder. To view a copy of this licence, visit http://creativecommons.org/licenses/by/4.0/.

\section{References}

1. Komori T, Scheithauer BW, Hirose T (2002) A rosette-forming glioneuronal tumor of the fourth ventricle: infratentorial form of dysembryoplastic neuroepithelial tumor? Am J Surg Pathol 26: $582-591$

2. Anyanwu CT, Robinson TM, Huang JH (2020) Rosette-forming glioneuronal tumor: an update. Clin Transl Oncol 22:623-630 
3. Yang C, Fang J, Li G, Li S, Ha T, Wang J, Yang B, Yang J, Xu Y (2017) Histopathological, molecular, clinical and radiological characterization of rosette-forming glioneuronal tumor in the central nervous system. Oncotarget 8:109175-109190

4. Wilson CP, Chakraborty AR, Pelargos PE, Shi HH, Milton CK, Sung S, McCoy T, Peterson JE, Glenn CA (2020) Rosette-forming glioneuronal tumor: an illustrative case and a systematic review. Neuro-oncol Adv 2. https://doi.org/10.1093/noajnl/vdaa116

5. Morassi M, Vivaldi $\mathrm{O}$, Cobelli M, Liserre B, Zorzi F, Bnà C (2020) A multifocal glioneuronal tumor with RGNT-like morphology occupying the supratentorial ventricular system and infiltrating the brain parenchyma. World Neurosurg 133:196-200

6. Sekar A, Rudrappa S, Gopal S, Ghosal N, Rai A (2019) Rosetteforming glioneuronal tumor in opticochiasmatic region-novel entity in new location. World Neurosurg 125:253-256

7. Al Krinawe Y, Esmaeilzadeh M, Hartmann C et al (2020) Pediatric rosette-forming glioneuronal tumor of the septum pellucidum. Child's Nerv Syst 36:2867-2870

8. Hamauchi S, Tanino M, Hida K, Sasamori T, Yano S, Tanaka S (2019) Spinal rosette-forming glioneuronal tumor: a case report. Medicine 98:e18271. https://doi.org/10.1097/MD. 0000000000018271
9. Collin A, Adle-Biassette H, Lecler A (2018) Rosette-forming glioneuronal tumor of spinal cord. World Neurosurg 119:242-243

10. Matyja E, Grajkowska W, Nauman P, Ozieblo A, Bonicki W (2011) Rosette-forming glioneuronal tumor of the fourth ventricle with advanced microvascular proliferation-a case report. Neuropathology 31:427-432

11. Johnson M, Pace J, Burroughs JF (2006) Fourth ventricle rosetteforming glioneuronal tumor. Case report. J Neurosurg 105:129-131

12. Medhi G, Prasad C, Saini J, Pendharkar H, Bhat MD, Pandey P, Muthane Y (2016) Imaging features of rosette-forming glioneuronal tumours (RGNTs): a series of seven cases. Eur Radiol 26:262-270

13. Gaudino S, Quaglio FR, Schiarelli C, Martucci M, Tartaglione T, Gualano MR, di Lella GM, Colosimo C (2012) Spontaneous modifications of contrast enhancement in childhood non-cerebellar pilocytic astrocytomas. Neuroradiology 54:989-995

Publisher's Note Springer Nature remains neutral with regard to jurisdictional claims in published maps and institutional affiliations. 